\title{
Riesgos y morfología urbana: ciudades en planicies y laderas
}

\author{
Elsa Lily Caballero Zeitún y \\ Celina Michelle Sosa Caballero
}

\section{Resumen}

Hoy en día podemos definir con mayor solvencia el concepto de riesgo urbano como la exposición a sufrir daños en vidas y medios de vida por la alta densificación poblacional y de infraestructura de los espacios socialmente construidos, cuyos detonantes pueden ser amenazas naturales 0 antrópicos. Las acciones humanas son las que magnifican la severidad de los daños a la sociedad, siendo los patrones de uso del suelo factor de riesgo que determina la pérdida de los bienes de vida de la población.

Periódicamente las ciudades de Honduras enfrentan desastres, situación que motiva el estudio sistemático de los riesgos urbanos, con el tema riesgos y morfología urbana: ciudades en planicies y laderas, interesa avanzar en la exploración del tipo de emplazamiento de la ciudad en relación a amenazas hidrometeorológicas, como una contribución con los criterios de la planificación urbana sostenible.

Palabras clave: Gestión de riesgos. Morfología urbana. Planificación urbana.

\begin{abstract}
Today we can advance define the concept urban risk as exposure to damage in lives and livelihoods by the high density of population and infrastructure of socially constructed spaces, whose triggers can be natural or man-made threats, where human actions are those that magnify the severity of the damage to society, being the land use patterns of risk factor which determines the loss of the goods of life of the population.

Periodically the cities of Honduras face disaster situation that motivates the systematic study of urban risks, themed Risks and Urban Morphology: cities in plains and slope, interested further explore the type of location of the city in relation to hydrometeorological as a contribution to the criteria of sustainable urban planning.
\end{abstract}


Keywords: Risk management; Urban Morphology; Urban Planning.

Elsa Lily Caballero Zeitún (zeitunlily@yahoo.com), Maestría en Gestión Social Urbana, UNAH. Celina Michelle Sosa Caballero (sosacelina@yahoo.com), FACES, UNAH. 


\section{INTRODUCCIÓN}

Amenazas antrópicas y naturales desencadenan desastres cada vez más severos en los asentamientos humanos, el crecimiento de las ciudades hace que cada vez sea mayor el número de personas y bienes afectados, por lo que progresivamente se introduce la gestión del riesgo en la planificación urbana o municipal.

Estudio realizados sobre riesgos urbanos coinciden en que los factores que potencian los daños por amenazas naturales particularmente las de origen hidrometeorológico, están asociadas a la forma en que se da el crecimiento urbano caracterizado por la segregación residencial, la fragmentación social y territorial, la profundización de la pobreza incremento de la vulnerabilidad y la ausencia de procesos de planificación urbana sistemática que produce la ciudad formal y la informal.

Del enfoque de gestión del riesgo, acuñado por la Red de Estudios Sociales en Prevención de Desastres, nos interesa destacar la importancia de la recurrencia de pequeños eventos de desastre, normalmente sólo se recupera la importancia de los grandes eventos de desastre, los de menor envergadura que ocurren con mayor frecuencia e igualmente provocan serios daños a las familias afectadas no tienen la misma cobertura. "Lo que interesa destacar, en primer lugar, es que la larga historia de desastres mayores ha ocultado la existencia de una serie de eventos de menor envergadura que ocurren cotidianamente en la ciudad y que no han sido objeto ni de estudios ni de programas de prevención sostenidos, y que junto al cuadro de riesgo de terremotos están los derrumbes, deslaves e inundaciones" (Lungo et al, 1996: 12).

Como sociedad todavía no estamos en condiciones de prevenir los grandes eventos que causan daños masivos (Haití 2010, Chile 2010, Japón 2011,) pero si estamos en condiciones de hacer cambios sustantivos en la forma de ocupación del suelo urbano para resistir mejor y prevenir los pequeños pero recurrentes eventos de desastre. El reto es corregir las intervenciones que generan riesgos, desde los sistemas constructivos, la ocupación de zonas de riesgo, la ausencia 0 debilidades de los sistemas de defensa civil, la anarquía en la ocupación del suelo, la deforestación, la desestabilización de suelos y taludes, la impermeabilización de suelos por edificaciones y carpetas asfálticas.

"Los procesos de ocupación territorial y de producción urbana, los patrones de uso del suelo, la falta de regulaciones para la construcción y los severos déficits y obsolescencia de la infraestructura y los servicios básicos, combinados 
con el crecimiento poblacional y de la pobreza urbana aumentan la presión sobre los recursos ambientales, exponiendo a una proporción cada vez más creciente de la población de las ciudades a enormes riesgos ambientales. Lo importante en la cuestión es que la generación de riesgos ambientales urbanos tiene un alto componente de participación humana, lo que platea la posibilidad de prevenirlos y evitarlos siempre y cuando se modifique la relación entre el ser humano y la naturaleza, y se formulen políticas urbanas que incorporen la prevención de los riesgos ambientales" (Lungo et al, 1996: 32).

Una relación que no ha sido lo suficientemente profundizada en la planificación y riesgo urbano es la importancia que adquiere el emplazamiento físico de la ciudad y las intervenciones urbanas, no en el sentido de volver al análisis puramente físico de los riesgos, etapa superada, sino en la interacción de factores físicos y sociales. Lo específico del emplazamiento de la ciudad y las formas de ocupación del suelo son determinantes en la severidad de los daños. Los daños por inundaciones, derrumbes, deslizamientos, sismos, tienen una estrecha relación con las capacidades de técnico políticas de gestión de la ciudad y la capacidad de sus ocupantes en el uso de tecnologías en la construcción social del espacio.

Al considerar el tipo de emplazamiento físico de la ciudad, es necesaria la diferenciación a partir de una tipología básica de ciudades en laderas y ciudades en planicie, el comportamiento de los fenómenos detonantes de desastres son diferentes según el emplazamiento de la ciudad, lo que se requiere de tratamientos técnicos y normativos diferentes que la estandarización de riesgo no ayuda a superar.

Normalmente, la planificación urbana parte de un diagnóstico físico de la ciudad para la zonificación, pero este instrumento hace referencia al uso funcional del espacio y no necesariamente a las adecuaciones normativas y tecnológicas que se derivan del tipo de emplazamiento, la premisa es que la tecnología, en este caso constructiva, resuelve los problemas de intervención según las características del territorio, sin embargo, la realidad de las ciudades es otra, las tecnologías constructivas en sí mismas no han resuelto las pérdidas del stock urbano frente a los desastres, "las tendencias recientes indican que los avances en la prevención de riesgos son positivas en la disminución de pérdidas humanas pero no está sucediendo lo mismo con los bienes de vida de la población, incluyendo en estos, las viviendas, menajes, instalaciones públicas y privadas en servicios y producción" (EIRD/ONU, 2011).

El tipo de emplazamiento físico de los asentamientos humanos es defini- 
tivo en cuanto a la exposición a riesgos y la diferencia está dada por la ubicación y emplazamiento, la historia geológica, las zonas climáticas, el tipo de suelo, los accidentes geográficos y la altitud. La población se asienta sobre un espacio construido único sobre valles, montañas, costas, humedales, etc., por lo que el riesgo de desastre dependerá de los procesos de ocupación del suelo con propósitos urbanos.

Por ejemplo, uno de los elementos de la morfología urbana es la traza, que supone tomar en cuenta la topografía del terreno, criterios técnicos de trazado de calles cuyo sentido de accesibilidad de prioridad a la seguridad humana, la traza orgánica para suelos escarpados, minimiza la erosión, la traza ortogonal o reticular, en planicies pueden ser diseñadas para la evacuación de aguas en casos de inundación y no solo para uso vehicular; trazas adaptadas a los suelos de bajo nivel freático de zonas costeras que no obstaculizan los cursos de aguas.

El objetivo del análisis de los riesgos y la morfología urbana es destacar la importancia del tipo de emplazamiento frente al riesgo de inundación. El documento se organiza en tres partes, la primera, trata sobre una tipología básica de emplazamiento básico de la ciudad; en la segunda, se hace el análisis de ciudades en laderas donde se toma el caso de la ciudad de Tegucigalpa, por estar emplazada en una cadena de laderas y posee un nivel de avance importante en cuanto a investigaciones especializadas; la tercera, analiza las ciudades en planicies, donde se toma el caso de la ciudad de Valle de Ángeles, emplazada en una meseta y la Ciudad de Tela, en una planicie costera y humedal, seleccionadas básicamente por el conocimiento en terrenos de ambos casos y se concluye con una discusión sobre las particularidades de la morfología y los riesgos urbanos según el tipo de emplazamiento y que pueden contribuir a mejorar los procesos de planificación urbana con enfoque de gestión de riesgos.

\section{EL TIPO DE EMPLAZAMIENTO DE LA CIUDAD}

Según sean las características del territorio bajo estudio, interesa determinar por topografía el tipo de emplazamiento de la ciudad el cual se distingue por una superficie de grandes áreas escarpadas, modeladas por el sistema de cordilleras y montañas y en menor medida la superficie plana de las planicies del norte y Pacífico.

Las zonas de montaña que se estructuran a partir de tres cordilleras, del norte, central y del sur. Las tierras altas del interior comprenden una superficie del $82 \%$ del total nacional. Las montañas y los cerros se encuentran disectadas en el 
occidente del país (Pineda Portillo, 1997: 51).

La mayor parte de los asentamientos humanos están emplazados sobre laderas o cadenas de laderas (Mapa 1) que según el dato del PATH en el 2001, significaba un total de 21,330 asentamientos sobre laderas.

\section{Mapa 1: Asentamientos humanos en laderas}

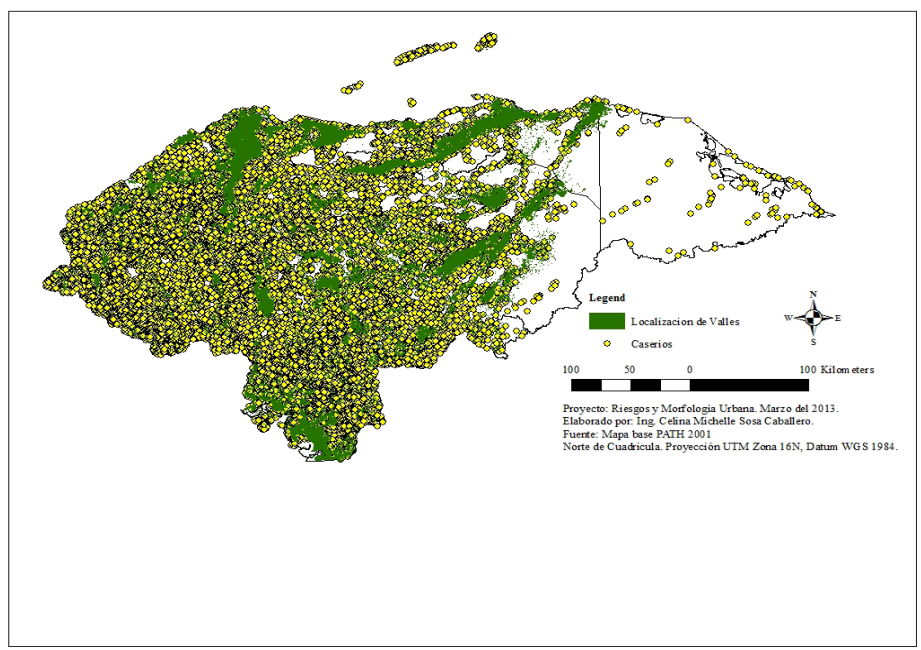

Las planicies de Honduras, compuestas por 52 estructuras entre altiplanos, planicies costeras, humedales, valles, llanos y mesetas entre cadenas montañosas donde se alojan unas 5,293 ciudades (Mapa 2). Según clasificación geomorfológica el país cuenta con las planicies costeras del norte y del Pacífico: las del norte, comprenden las tierras bajas de la costa norte abarcan una superficie de $16 \%$ del territorio nacional y se caracterizan por tener tierras planas, clima tropical caliente y lluvioso. Las precipitaciones llegan hasta los 2000 mm., y en algunas áreas las temperaturas son también elevadas, mayores a los 24 grados centígrados. Las tierras bajas del pacifico comprende un área de $2 \%$ del total del país, con una extensión del litoral costero de $133 \mathrm{~km}$ lineales. Con suelos aluviales y de planicie costera susceptibles de regadío. Clima propio de sabana, tropical lluvioso seco. Constituye una pequeña unidad fisiográfica que bordea el golfo de Fonseca. (Pineda Portillo, Noé, 1997: 49 y 72). 


\section{Mapa 2: Asentamientos humanos en planicies}

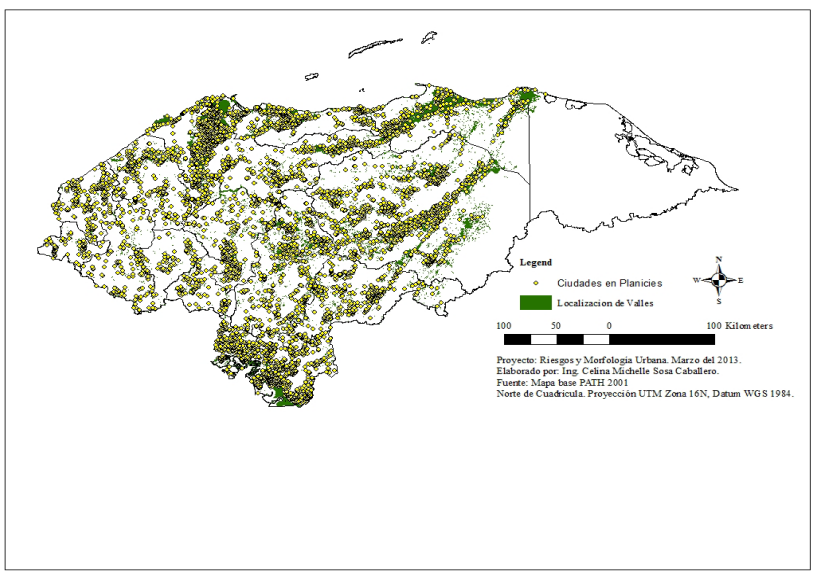

\section{CIUDADES EN LADERAS}

El concepto de ladera urbana se ha definido como "la forma de ocupación del suelo sobre un accidente geográfico 0 una cadena de accidentes geográficos con declives, con un uso intensivo en vivienda equipamiento urbano e infraestructura económica y social, susceptible de presentar movimientos de subsidencia o colapso por la incidencia y combinación de factores internos y externos, que constituye un riesgo de desastre para sus ocupantes y sus bienes de vida" (Caballero Zeitún, 2011).

En el caso de las laderas urbanas los comportamientos de los fenómenos hidro meteorológicos desencadenan en derrumbes, deslizamientos y colapsos por saturación de agua, activación de fallas y cizallas, condicionadas estructuralmente para el caso de Honduras por la topografía, la zona climática y la geomorfología.

Este primer nivel de análisis (escala 1:2,600.000) nos determina la primera clasificación de la tipología de emplazamiento (laderas o planicies) de los pueblos y ciudades, a los que agregándole la ubicación en la latitud tropical (16 grados latitud norte) con vientos alisios NE, condiciona los riesgos de inundación y deslizamientos. 


\subsection{Ciudades en laderas, el caso del Distrito Central de Honduras}

En el año 2002, la Cooperación Japonesa realizó, probablemente, uno de los estudios más completos que existen en el país sobre riesgos por inundación y deslizamientos para el Distrito Central de Honduras. "El estudio reveló que el $30 \%$ del área objeto para la prevención de desastres está ocupado por áreas bajo amenaza de inundación y deslizamiento y el $15 \%$ de la población total vive en estas áreas peligrosas. El objetivo del Plan Maestro es minimizar los daños y evitar cualquier pérdida de vida humana" (JICA, 2002).

El área de estudio para la prevención de desastres del área urbana de Tegucigalpa es de $105 \mathrm{~km}^{2}$ en una elevación entre 900 m y 1,400 msnm. Por las formación geológica (rocas sedimentarias de la edad Cretácea como base, rocas volcánicas de la edad Terciaria que cubren las rocas Cretáceas y rocas volcánicas y rocas sedimentarias del Cuaternario que cubren las rocas Terciarias) y la precipitación anual (varía entre $800 \mathrm{~mm}$ y 1,500 mm y el promedio del área es de 1,000 mm/año, siendo mayor en la cuenca del río Guacerique, el río Grande y en la montaña La Tigra $(1,200 \mathrm{~mm}$ hasta $1,400 \mathrm{~mm})$ y en el área sudeste, las cuencas del río Sabacuante y el río Canoas es menor $850 \mathrm{~mm}$. Los ríos de la ciudad sirven como desagües al aire libre para la descarga de aguas servidas sin tratar, proveniente de todo tipo de usos de origen doméstico, institucional, comercial e industrial El continuo desagüe de aguas servidas sin tratar provocó la contaminación del lecho de los ríos donde la superficie del lecho está cubierta virtualmente por fango de las aguas servidas más que por el suelo natural, especialmente en los tramos del río con baja velocidad de flujo. Para el análisis de riesgo de deslizamiento de tierra, se observó los patrones de deslizamientos de tierra y derrumbamiento de talud que se observaron durante el huracán Mitch (se examinaron con precisión las fotografías aéreas con la escala de $1 / 10,000$ y orto fotos para interpretar las características topográficas de los deslizamientos de tierra), los que se identificaron según las características topográficas típicas de deslizamientos de tierra y se definieron como bloques susceptibles de deslizamientos de tierra. Esos deslizamientos en el área objeto se clasificaron de acuerdo con los patrones geológicos que los provocaron (JICA, 2002). 
Mapa 3: Amenaza de inundación ciudad de Tegucigalpa

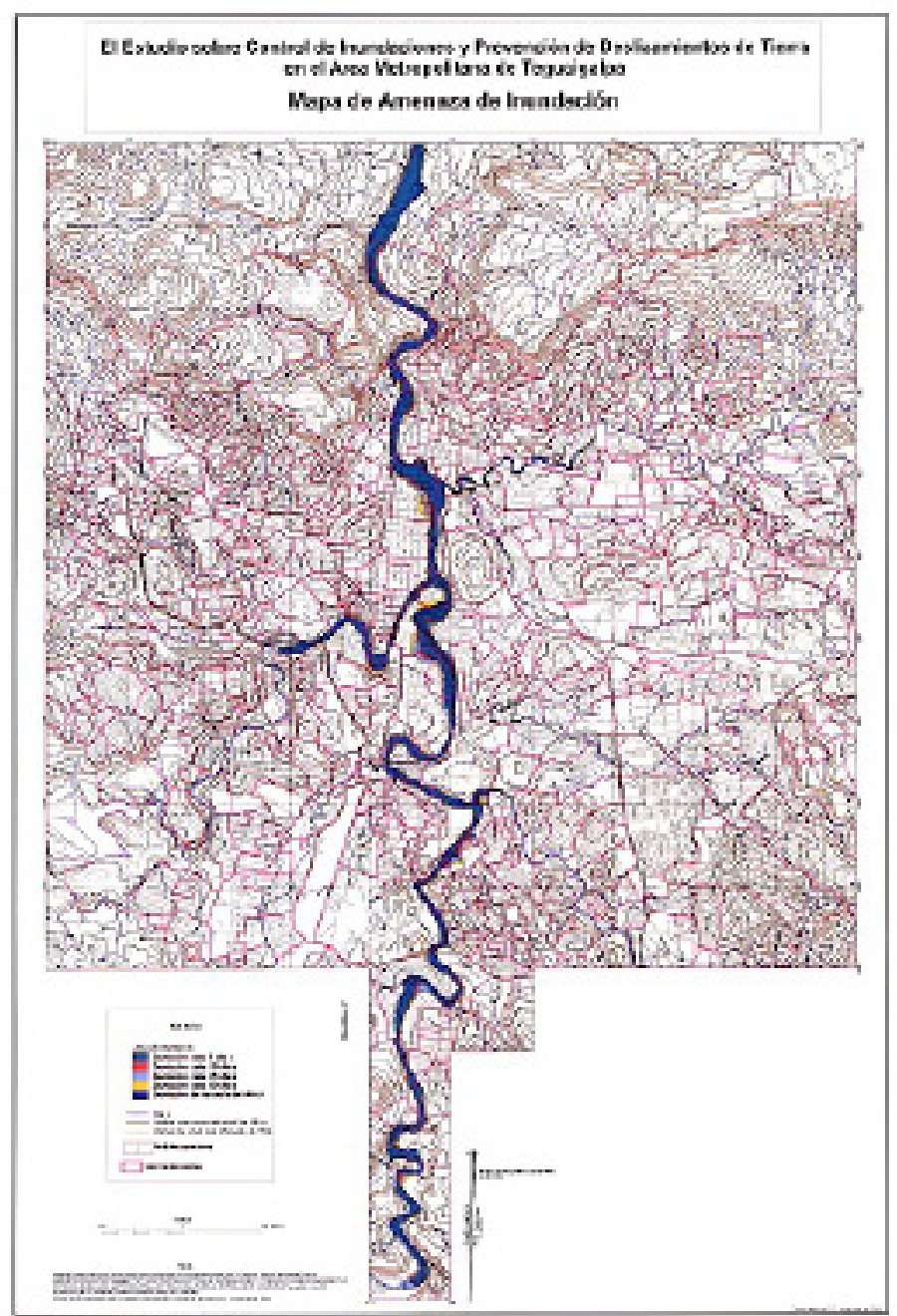

Fuente: JICA, 2002

El estudio comprende diversos tópicos para el análisis físico y socioeconómico de los territorios y poblaciones bajo condiciones de riesgo en la ciudad capital. Los temas de análisis son la geología, topografía, hidrología, precipitación, medio ambiente natural, usos del suelo, características económicas y sociales de la población análisis en una escala de 1:40.000 (Mapa 3) para el diagnóstico y propuesta. 
Otro esfuerzo importante en los procesos de planificación urbana con enfoque de riesgo consiste en un esfuerzo conjunto entre la municipalidad del Distrito Central, COPECO, CARE y la Unión Europea, con el proyecto "Manejo de riesgos en Tegucigalpa". Que tomando en cuenta la percepción de riesgo de los habitantes se elaboró 51 mapas de riesgos por barrios y colonias con escalas entre 1:2.000 a 1:3.500 (Mapa 4) y 8 mapas sectoriales en escalas de 1:10.000 a 1:20.000 (Mapa $5)$.

Mapa 4: Amenazas Bo. Abajo, Tegucigalpa

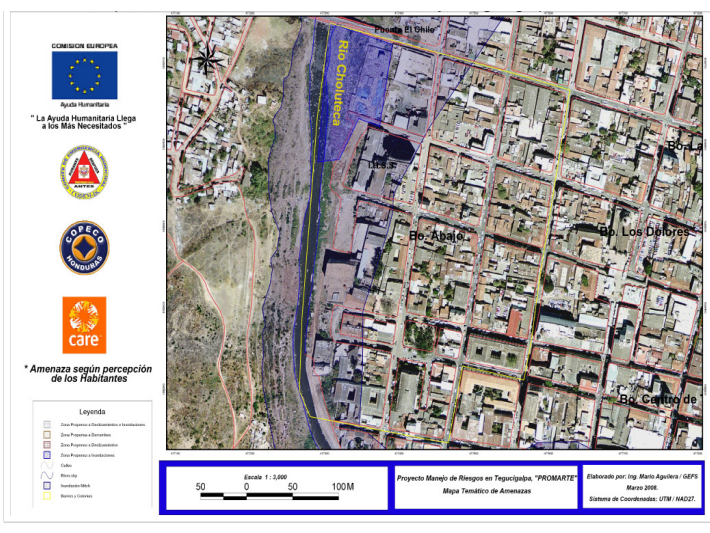

Mapa 5: Mapa sectorial de amenazas

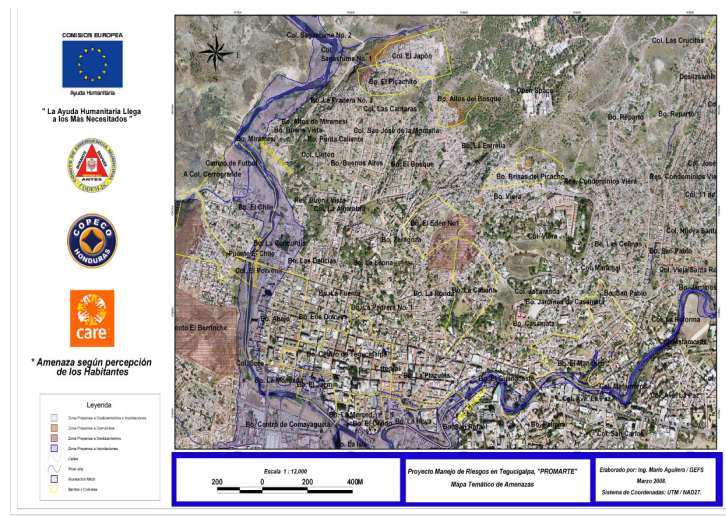

Fuente: Municipalidad del Distrito Central, COPECO, CARE y la Unión Europea, 2008 
Finalmente, el mapa multi amenazas (inundaciones y movimiento de laderas) del municipio del Distrito Central, República de Honduras, en una escala de 1:30000, está articulado a una ordenanza de certificación de profesionales de la construcción en gestión de seguridad y riesgos, según Acta No. 004 del Consejo Municipal del Distrito Central, del 10 de Marzo del 2011. El instrumento pasa a

formar parte de los requisitos para otorgar permisos de construcción.

El caso de la ciudad de Tegucigalpa ilustra sobre las variables a estudiar (topografía, precipitación, geología, usos de suelo, cursos y estado de cuerpos de agua, población expuesta y formas de ocupación del suelo) y las escalas de análisis para los diferentes niveles de intervención (ciudad, región o zona y barrios 0 localidad) para la gestión de riesgos en la ciudad.

\section{CIUDADES EN PLANICIE}

La planicie es un terreno llano, porciones del territorio con ninguna o pocas variaciones de altura respecto del nivel medio del mar, que pueden ser de grandes extensiones o pequeñas mesetas.

Para el tema que nos ocupa, las ciudades en planicie de manera general las definimos como aquellos asentamientos humanos que su emplazamiento es sobre un terreno principalmente plano con una cobertura intensiva de edificaciones, su forma de ocupación para usos urbanos puede ser planificada o espontanea.

La sociedad ha privilegiado las planicies como las mejores áreas del territorio para el desarrollo de las actividades humanas, pero también son muchos los registros de inundaciones de ciudades por desbordamientos violentos o crecidas no violentas del nivel de las aguas de afluentes próximos a los asentamientos.

Las principales intervenciones se relacionan a tecnologías de control de inundaciones, sin embargo, en los procesos de expansión urbana que generalmente terminan por ser espontáneos no siempre se toman en cuenta sistemas constructivos seguros, a pesar de que existe una variedad de tecnologías, por ejemplo, la construcción sobre polines, tecnologías para asegurar la permeabilización del suelo, el uso de inclinaciones que permitan la evacuación de aguas, etc.

A pesar de que el riesgo de inundación es generalizado para todo el país, no se identificó literatura que analice este riesgo en ciudades en planicie, el ámbito de análisis es municipal. A manera de ejemplo se analizan dos casos, en una meseta la ciudad de Valle de Ángeles y en planicie costera y humedal, la ciudad 
de Tela. La información cartográfica se ha capturado con tres programas, Google Earth, para ubicar las coordenadas de localización de las ciudades en estudio, el programa Quantungis, para levantar los rasgos principales de la morfología (traza urbana, cursos de agua, ocupación del suelo) y Arc Map para la edición de mapas.

\section{1 Meseta caso de la ciudad de Valle de Ángeles}

El municipio está en una zona de transición entre bosque latifoliado y bosque de pino, parte del territorio está dentro de la zona protegida Parque Nacional "La Tigra", reserva de biodiversidad y zona productora de agua, asentado sobre un terreno escarpado, con una altitud promedio de $1400 \mathrm{msnm}$, temperaturas promedio de 16 y 20 grados centígrados con dos estaciones al año la seca y lluviosa, esta última con una duración promedio de ocho meses de lluvia y una precipitación promedio anual entre 1500 a $2500 \mathrm{~mm}$.

"Este municipio presenta una zona montañosa, un área de pie de monte y un valle estrecho que es afectado en materia de inundación por el río Soledad y sus afluentes, las quebradas Agua Dulce, Carrizal, Jutes, San Francisco y Agua Amarilla. Estos afluentes se adentran al casco urbano Valle de Ángeles, el cual está expuesto a severas inundaciones asociadas con fenómenos geológicos de arrastre de piedras y sedimentos por represamiento y rompimiento de derrumbes en los ríos. Los problemas en el casco urbano de Valle de Ángeles están relacionados con la destrucción de casas, la inestabilidad de los ríos con la posibilidad de cambio del cauce, la destrucción de puentes y alcantarillas, como en el Edén y la incomunicación con Tegucigalpa y comunidades locales" (CATIE, 2002).

Para el análisis de riesgo por inundación en la ciudad de Valle de Ángeles (Mapa 5) se ha levantado el trazo de la ciudad, en una escala de 1:21.000, identificado el área comprendida entre dos quebradas que drenan al río La Soledad, al suroeste la quebrada Agua Dulce y noreste quebrada Agua Amarilla.

Se clasifican tres tipos de zona para la gestión del riesgo (Mapa 6): mitigación, área de mayor densificación de construcción y debe ser intervenida con obras para corregir las formas de ocupación del suelo; prevención, menor densificación constructiva, se pueden tomar medidas para futuras construcciones y área de preservación, donde aún no se ha construido y debe ser sujeto de una normativa estricta para orientar futuras intervenciones. 
Mapa 6: Riesgo por inundación, ciudad de Valle de Ángeles

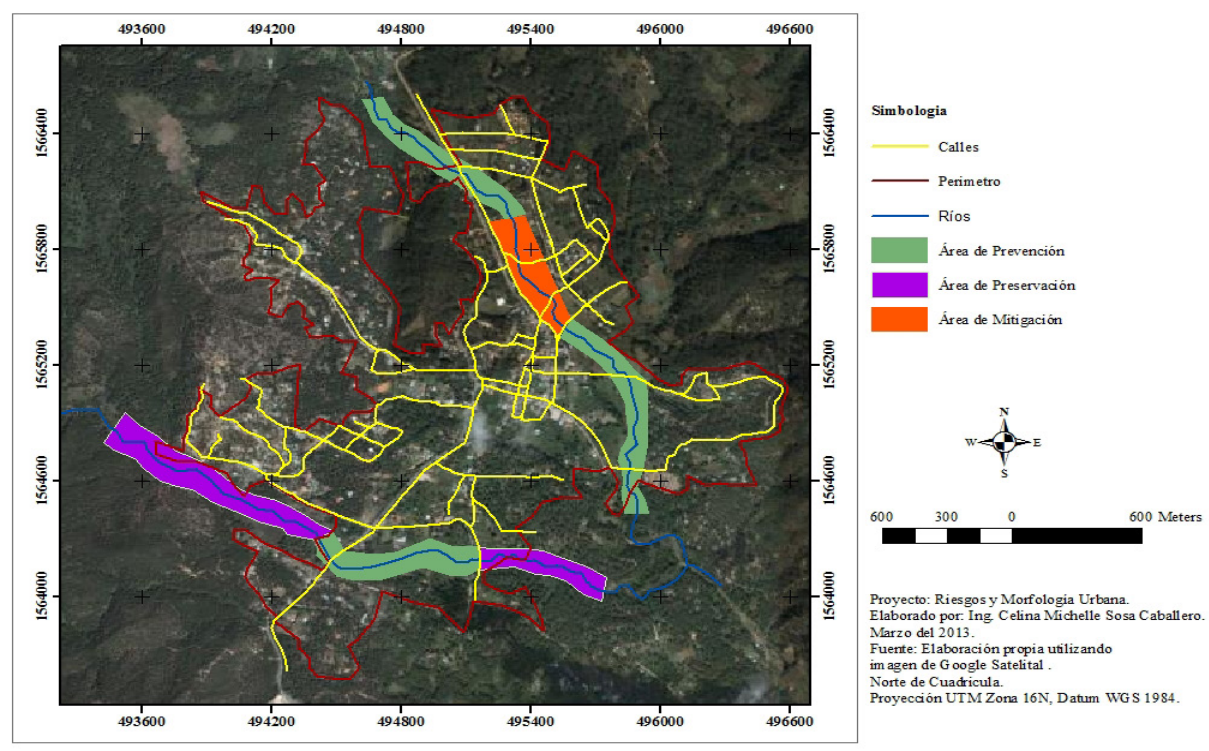

Para los diferentes tratamientos el plano de la ciudad se segmenta por micro sistemas (Figura 1) determinados por el curso de las quebradas según los grados de densificación y patrones de ocupación del suelo.

Figura 1: Zonificación de riesgos de Valle de Ángeles

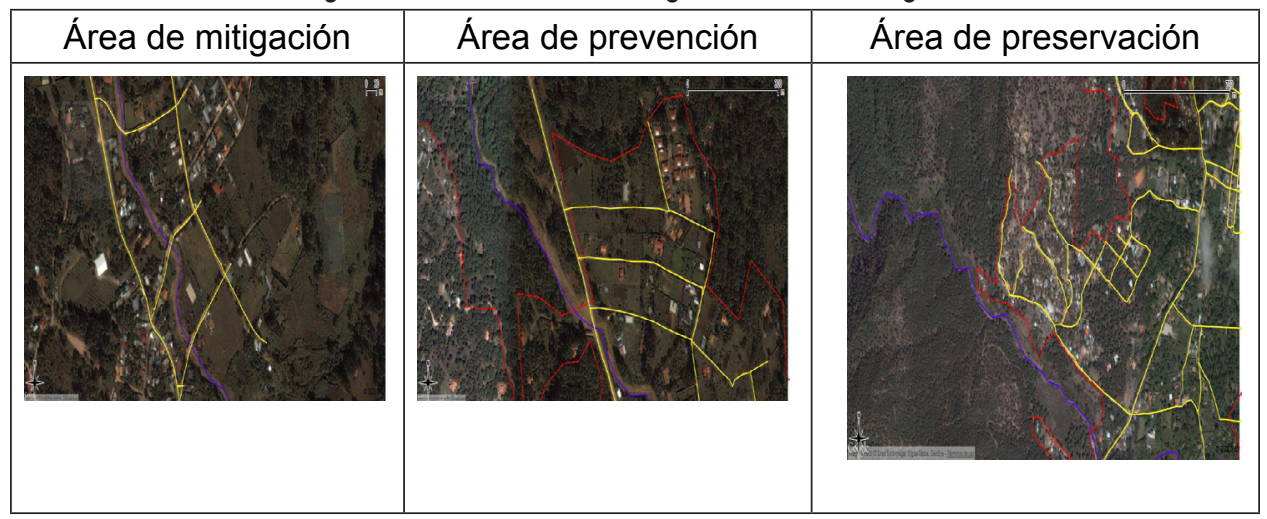

Fuente: Proyecto Morfología y Riesgo Urbano, marzo 2013. 


\subsection{Planicies costeras y humedales, el caso de la ciudad de Tela}

La ciudad se ubicada al sureste de la bahía de Tela en el Mar de las Antillas, una franja costera de aproximadamente $35 \mathrm{~km}$ de playa donde se encuentran las playas de San Juan, El Triunfo, La Ensenada, Tela, entre áreas de humedales y lagunas, al oeste el Parque Nacional Jeannette Kawas o Punta Sal (78.400 ha.); la Laguna Quemada o de los Micos y Laguna el Diamante; al este el Parque Nacional Punta Izopo (18,500 ha.) y la Laguna Negra y al norte el Jardín Botánico Lancetilla (1.681 ha. de bosque) y el refugio de vida silvestre Texíguat (16.000 ha. de bosque). La ciudad está regada por los ríos Hiland, La Lancetilla, Tela y La Esperanza. Los meses más lluviosos son de octubre a diciembre, con un promedio anual total de lluvia 2,900 mm.

Inicialmente la ciudad se desarrolla por la actividad portuaria de las compañías bananeras y posteriormente se convierte en destino turístico (playa y ecoturismo), actuantemente se estima una población de 24 mil habitantes.

Para la clasificación de riesgos (Mapa 7) se usa el mismo criterio de la ciudad de Valle de Ángeles. El mapa de Tela está en una escala de 1:37.000

\section{Mapa 7: Riesgo de inundación, ciudad de Tela}

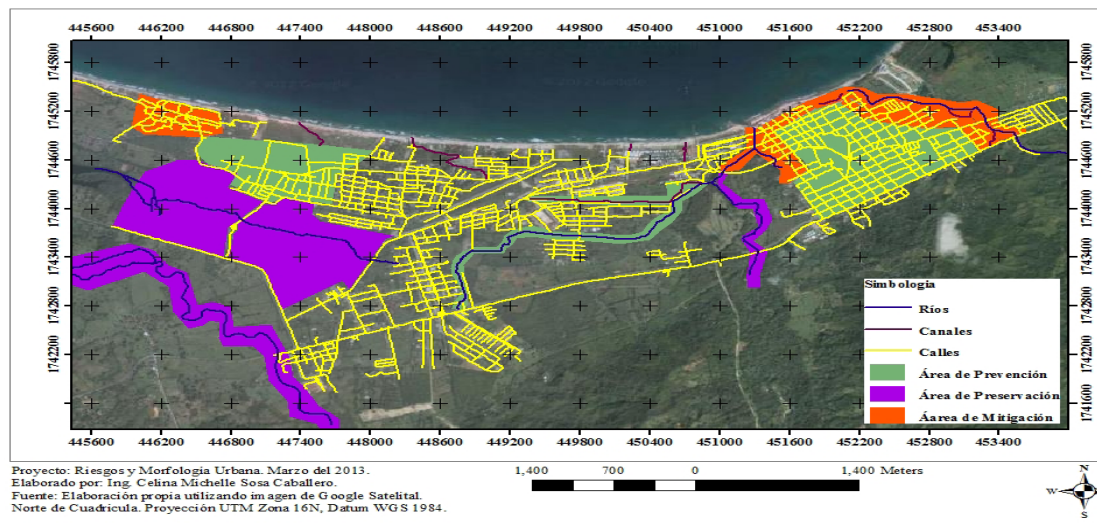

Para los diferentes tratamientos el plano de la ciudad (Figura 2) se segmenta por micro sistemas determinados por el curso de los ríos, altitud y tipo de suelo, grados de densificación, patrones de ocupación y disposición de sistemas de alcantarillado. 
Figura 2: Zonificación del riesgo

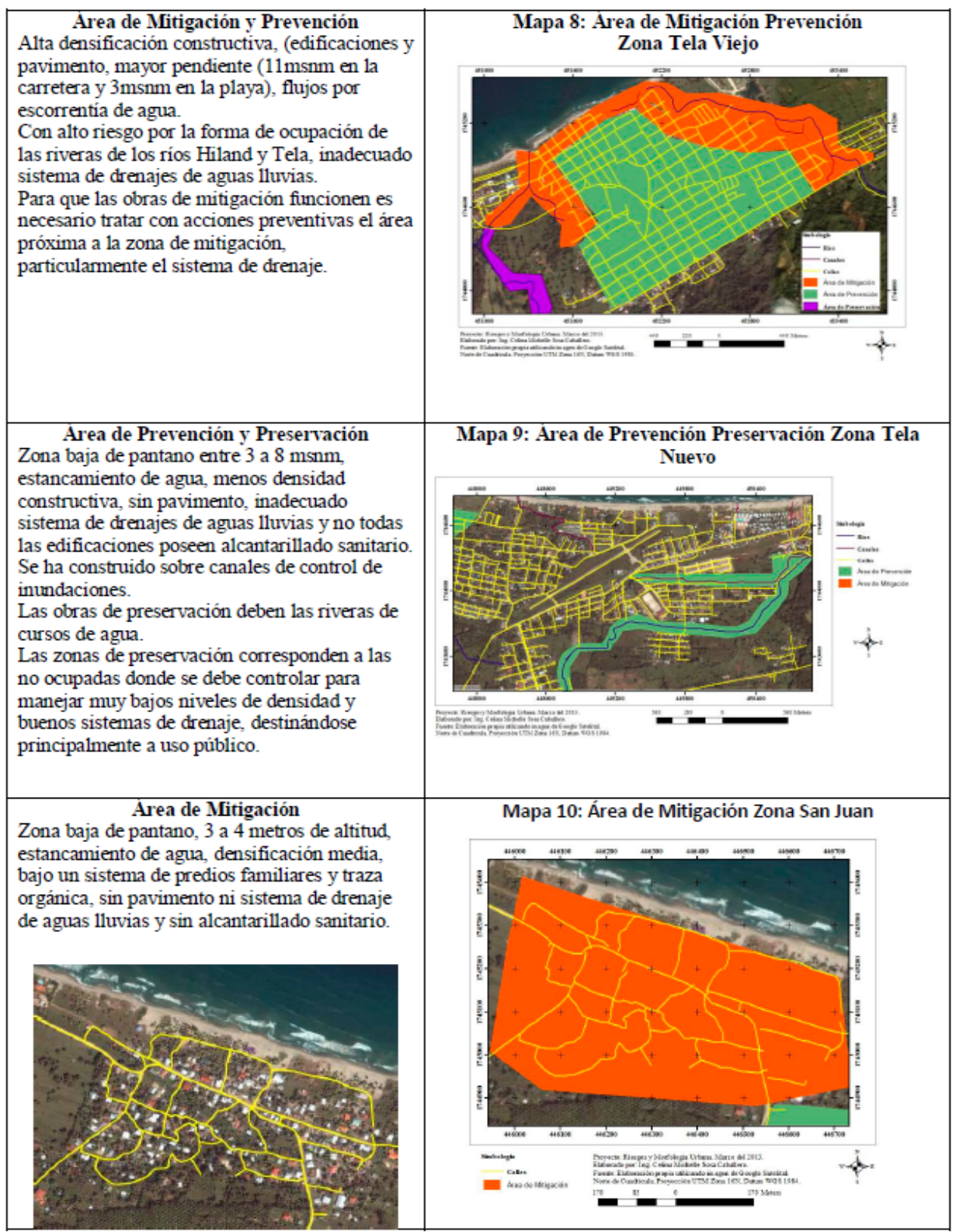




\section{RIESGOS URBANOS Y EL TIPO DE EMPLAZAMIENTO DE LA CIUDAD}

Aunque el tamaño de la ciudad es un factor importante en los procesos de gestión urbana, la complejidad del problema de los riesgos está más asociada a los microsistemas identificables dentro de la mancha urbana, donde se tienen niveles de análisis y categorías de clasificación de riesgos.

Los niveles de análisis de las diferentes variables corresponden a las siguientes escalas (Figura 3).

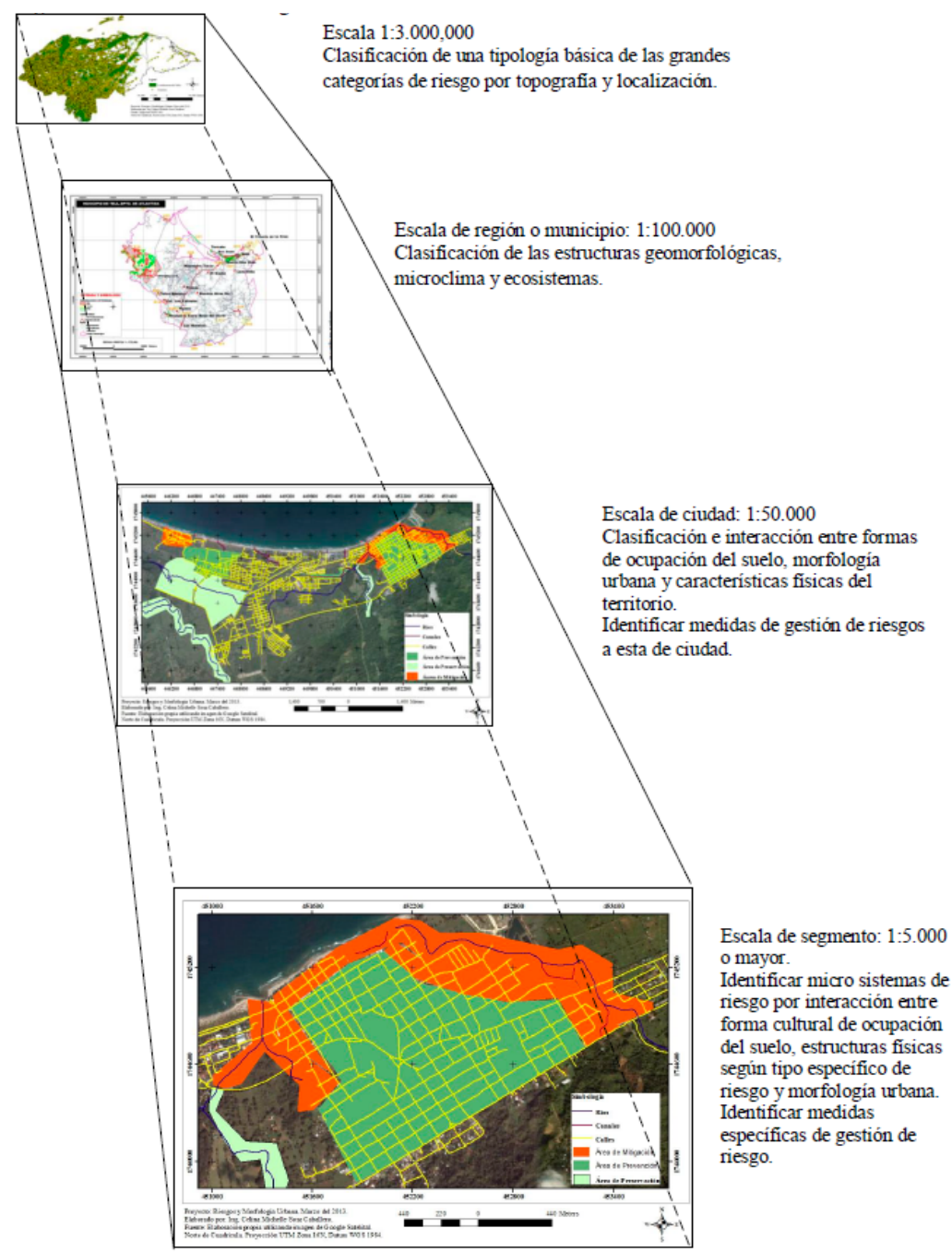

Fuente: Proyecto Morfología y Riesgo Urbano, marzo 2013 
Al incorporar la gestión del riesgo a la planificación urbana, el propósito fundamental y el enfoque esencial es la actuación antes de que sucedan los daños, por lo que las categorías de clasificación del riesgo adquieren connotaciones e interacciones diferentes según sea el emplazamiento, para lo que, primero, es necesario diagnosticar el ecosistema de la ciudad donde la variable central es la forma de ocupación del suelo, segundo identificar segmentos de la ciudad por microsistemas que se pueden diferenciar en la mancha urbana, para diseñar las medidas específicas de intervención como parte del proceso de la gestión del riesgo. En los dos ejemplos usados podemos diferenciar:

a. Ladera urbana: El sistema de laderas de Tegucigalpa presenta dos categorías de riesgo relacionados a fenómenos hidrometeorológicos:

Zona escarpada constituida por una cadena de laderas de alta densificación constructiva y densidad poblacional; con problemas de escorrentías por obstrucción de drenajes por asolvamiento y depósito de basura o la no existencias de estos; por tipo de suelo propenso a deslizamientos, recurrentemente activados por agua. Ocupación de riveras de cursos de ríos y quebradas propensas a inundación.

b. Planicie urbana: El sistema de meseta Valle de Ángeles en área de montaña. Los microsistemas identificables son determinados por los cursos de agua y el grado de densificación constructiva de las riveras de las quebradas.

c. Planicie urbana: El sistema de Tela, en área de playa y humedal, con microsistemas diferenciados por grados de densificación constructiva, tipos de traza urbana, donde la altitud y el tipo de suelo inciden de dos formas, por escorrentía o por estancamiento de agua. Donde las áreas de obras de mitigación no pueden estar disociadas de las áreas de prevención o preservación.

Los ejemplos ilustrados, ayudan a avanzar en el concepto de riesgo urbano ya que los niveles de desagregación requeridos serán según sean las características de la morfología urbana; el tipo de emplazamiento de las ciudades; las formas culturales de ocupación del suelo y los diferentes escenarios de densificación constructiva y poblacional que se puedan dar. Elementos indispensables para salvar vidas y medios de vida en los asentamientos humanos ya no como intervención bajo emergencia y ayuda humanitaria sino como parte de la matriz de planificación urbana. 


\section{BIBLIOGRAFÍA}

- Agencia de Cooperación Internacional del Japón (JICA) (2002). "El estudio sobre el control de inundaciones y prevención de deslizamientos de tierra en el área metropolitana de Tegucigalpa de la Republica de Honduras". Tegucigalpa, Honduras. (Versión digital).

- CINSA(2006). "Caracterización y planificación territorial para la gestión local de riesgos región CT-08, plan municipal de gestión de riesgos municipio de Tela departamento de Atlántida" Secretaría de Gobernación y Justicia. Tegucigalpa, Honduras. (Versión digital).

- CATIE (2002) "Plan de prevención y mitigación del municipio de Valle de Ángeles" (Informe impreso).

- Caballero Zeitún, Elsa Lily (2011). "El concepto de laderas urbanas". Taller Regional para Centroamérica, México y el Caribe "Cerrando la Brecha de Saneamiento". 18 a 23 septiembre 2011, Costa Rica. Acuerdo de Cooperación entre el Centro Nacional de Competencia en Investigación Norte-Sur (NCCR), La Oficina de Coordinación Regional de México, Centroamérica y el Caribe (JACS CCA RCO) y el PLATS-UNAH. Programa RP9.

- Diálogo Interamericano de Reducción de Desastres (1998). "Integrando la reducción de desastres en la esencia del desarrollo" (Diálogo II). Disponible en: http:www.oas.org.

- EIRD/ONU (2011). Informe de evaluación global sobre la reducción del riesgo de desastres (CAR 2011) Revelar el riesgo, replantear el desarrollo. Oxford, Reino Unido: Information Press.

- Fundación VIDA/UE-PRRAC D (2004). "Apoyo a la gestión municipal ambiental en 25 municipios de Honduras". Convenio acr/b7-3130/1b/99/0302 Diagnóstico ambiental municipal participativo y plan de acción. Disponible en: http://www. fundacionvida.org/uploaded/content/category/1643124930.pdf

- Lungo, Mario et al (1996). De terremotos, derrumbes e inundaciones. San Sal- 
vador, El Salvador: La RED, FUNDE.

- Lavell y Mansilla (2003). "Vocabulario controlado del CRID". Disponible en: http://www.crid.or.cr/crid/CD_VCD/DocsIndex.htm

- Pineda Portillo, Noé (1997). Geografía de Honduras. Tegucigalpa.

- Secretaría de Gobernación y Justicia (2003). Plan estratégico de desarrollo municipal. Municipio de Tela, departamento de Atlántida. Tegucigalpa, Honduras. (Versión digital). 